\title{
Influencia de la educación para el control del fósforo sérico realizada por enfermería a pacientes y sus cuidadores principales
}

\author{
Laura Hernández Moreno, Romy Judy Perinango, Eva Casanovas Izquierdo
}

Institut Mèdic Badalona. Diaverum Servicios Renales. Barcelona

\section{Introducción:}

La población renal que entra en el programa de hemodiálisis, es cada vez de edad más avanzada, esto conlleva a que estos enfermos renales arrastren una serie de pluripatologías y que frecuentemente necesiten, cada vez más, la figura de un cuidador principal que controle y se responsabilice de sus cuidados, en especial de su dieta, medicación. El objetivo de este trabajo es, demostrar la efectividad de la educación sanitaria en un centro no hospitalario a los pacientes y a sus cuidadores principales para conseguir disminuir los niveles de fósforo sérico. Se realiza a través de un estudio experimental cuantitativo de 20 meses de duración y con una muestra de 70 pacientes divididos en dos grupos aleatorios de 35 pacientes cada uno; un grupo control que no recibe educación adicional y un grupo estudio que recibe la educación específica para el control del fósforo sérico y su dieta; en este grupo se identifican los pacientes dependientes que requerirán de un cuidador, éste será el que reciba la educación. En ambos casos se utiliza como soporte material visual (fotografías y dibujos). Para la identificación del grado de dependencia del paciente se utiliza el Test Delta y para valorar la sobrecarga de atención sobre el cuidador principal se utiliza la escala de Zarit. Se obtiene como resultado que el $52.9 \%$ de nuestros pacientes son independientes; el $48.2 \%$, presentan una dependencia leve y el $4.3 \%$, presentan una dependencia moderada, sobre estos pacientes se realiza la educación con el soporte adicional del cuidador principal. Según la escala de Zarit el 33\% se consideran con sobrecarga leve, el tiempo de atención oscila entre 6 y 15 años; el 34\% con sobrecarga intensa, el tiempo de atención es de más de 15 años; el
$33 \%$ no se consideran sobrecargados siendo el tiempo de atención en este grupo menor de 5 años. El análisis de los resultados obtenidos pone de manifiesto una disminución significativa del nivel de fósforo sérico en el grupo estudio, consiguiendo que, el $21 \%$ de estos pacientes cuyo nivel de fósforo se encontraba por encima de $5.5 \mathrm{mg} / \mathrm{dl}$, lo redujera hasta los niveles normales recomendados $(3-5.5 \mathrm{mg} / \mathrm{dl})$. Concluimos que la implicación del personal de enfermería tanto en la educación continua del paciente y de sus cuidadores es necesaria y fundamental para conseguir unos resultados clínicos adecuados. Son los cuidadores principales, en especial, los que manifiestan sentirse satisfechos y reconfortados con el soporte recibido.

\section{Referencias Bibliográficas}

1. González M, Álvarez P, Vázquez J, Rodríguez M, Outeriño S, Manso B, Remigio M, Otero A, Graña J, Bañobre A, Estevez A.: efectividad de la intervención educativa en cuidadores de pacientes dependientes en diálisis y valoración de la carga. Revista de la sociedad española de nefrología 2005, 8 (2).

2. Andreo $L$, Moreno C.: Perfil y realidad social de los cuidadores principales de pacientes dependientes tratados con hemodiálisis. Nursing 2009, 27 (4): 54-65.

3. Andreo L, Moreno C, Julue M.: Características del cuidado familiar a pacientes dependientes en programa de hemodiálisis. Revista de la Sociedad Española De Enfermería Nefrológica 2011, 14 (1). 
4. Wilson Astudillo A, Carmen Mendinueta A.: Necesidades de los cuidadores del paciente crónico: guía de recursos sanitarios y sociales en la fase final de la vida en Guipuzcua. $7^{a}$ sección. - dfarmacia.com: Hiperfosfatemia,avances farmacológicos.

5. Muro Suesrun B, Pernaut Villanueva J, Urzainqui Laborda D.: Control del metabolismo del fósforo en la consulta prediálisis. Hospital Virgen del camino. Pamplona.

6. Alonso Latorre M, Blasco Pérez M.J, Castillo Hernández $A$, Cuevas Cámara $P$, Febrero $C$, López $S$, Quintana I, Romero S, Sancho M.T.: Influencia de enfermería en el control de la hiperfosfatemia. Instituto nefrológico Fresenius Medical care. Barcelona.

7. Sánchez I, Lorenzo V.: Hemodiálisis:¿Cuánto sabemos de los fármacos relacionados con el metabolismo mineral?. Sección de nefrología. Hospital Universitario de Canarias. Sta Cruz de Tenerife.

8. Rota L, Yuste $E$, Mañé N, García $E$, Marcet $M$, Marquina D, Ramírez N.: Diseño de una consulta de enfermería.Educación a los pacientes en hemodiálisis. Unidad de nefrología. Corporació Parc Taulí.

9. Arrojo A, Fernández A, Ruiz M.L, Fernández G, Sancho N.: La intervención enfermera:una garantía en el control de la hiperfosfatemia. Centro de diálisis Dialsan. Fresenius Medical care. Santander.

10. Villas A, Mañé $N$, Martínez G, Masip M, López M, Picazo L, Ponz E, Real J, Rocabert M, Valenzuela M.P, Yuste E.: Análisis del cumplimiento farmaco- lógico de los pacientes en hemodiálisis. Corporación Parc Taulí.

11. Mayor Iturburuaga J.M.: La alimentación en el enfermo renal, desde el punto de vista de enfermería ¿Cómo reforzar el cumplimiento?. Hospital Galdakao-Usansolo.

12. Cobo Sánchez J.L, Pelayo Alonso $R$, Sánchez Cano M.S, Alonso Nates R, Menezo Viadero R, Gándara Revuelta M, Rojo Tordable M, Cuadrado Mantecón M.E, Villa Llamazares C.: Repercusión del grado de dependencia de los pacientes en hemodiálisis sobre la carga de trabajo de enfermería. Unidad de hemodiálisis. Hospital Universitario Marqués de Valdecilla. Santander.

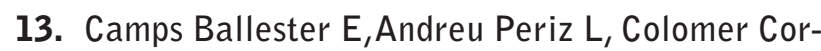
dinachs $M$, Claramunt Fonts $L$, Pasaron Alonso M.: Valoración del grado de autonomía funcional de pacientes renales crónicos según índices de barthel, lauton y baremo de ley de dependencia. Unidad de nefrología del consorcio hospitalario de Vic.

14. Ochoa Alfaro B.M, González Castillo $S$, Santesteban de la Concepción A, García Castillejo M.J, Barricarte Navarroe E, Zapatería Azcona C.: Análisis del grado de dependencia de pacientes en programa de hemodiálisis periódica en un hospital comarcal. Hospital Reina Sofía. Tudela.

15. Hernández Arribas V, Palacios García G, Rubio Gil F.J, Porras Montero G, Rodríguez Peña J, Uguet Canal M, Fernández Lucas M.: Evaluación del grado de dependencia de los pacientes en hemodiálisis mediante el test delta. 